\title{
Viscoelastic constitutive law based on the time scale of the mechanical phenomena
}

\author{
D. P. Pioletti ${ }^{1}$ \\ Ecole Polytechnique Federale de Lausanne (EPFL), Laboratory of Orthopedic Research \\ dominique.pioletti@epfl.ch
}

Experimental characterizations of biological soft tissues show that the stress-strain curves are sensitive to the strain rate at which they are performed and relaxation phenomena are present. Hence the strain rate is an important variable in the understanding of the mechanical behavior of soft tissues. Many of the constitutive models that have been proposed fail to properly consider this variable in large deformations. The development of a conceptual framework to test different viscoelastic constitutive laws will be presented. This framework has the advantage to satisfy a priori the thermodynamical restrictions and is valid for large deformations. In addition, the different mechanical contributions are separated according to the time scale of their effects. The obtained constitutive law takes into account the non-linear stress-strain curves with the strain rate as an explicit variable. As illustration of its ability to model the immediate, short time memory and long time memory contributions, the framework is used to identify mechanical tests performed on human ligaments. Good correlations are found between experimental and theoretical stress-strain curves. The identification results then in a realistic viscoelastic constitutive law. The developed viscoelastic law is proposed to model biological tissues presenting an important viscoelastic behavior.

\section{Mechanical behaviors according to their time scale}

The \index\{viscoelastic properties $\}$ of soft tissues play essential role in the kinematics and biomechanics of the human body. Intervertebral discs, as shock-absorption, can fulfill its function thanks to its viscoelastic properties e.g. Palmer and Lotz (2004). Ligaments or tendons' stiffness vary accordingly to the strain rate e.g. Danto and Woo (1993); Pioletti et al. (1999) allowing to obtain an optimal mechanical behavior between physiological joint laxity and stability. From a more general point of view, the mechanical behavior of soft tissues can be distinguished according to the time scale of their effects. Uniaxial tension tests performed on a human anterior cruciate ligament (ACL) are displayed on Figure 1.

It would be interesting to describe with the same constitutive law the contribution of the immediate, the \index\{short time memory\} and the \index\{long time memory\}. 


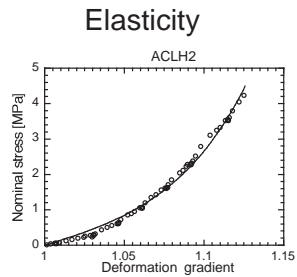

Time scale:

Immediate

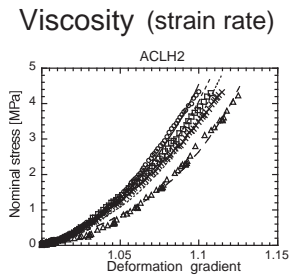

Short time memory

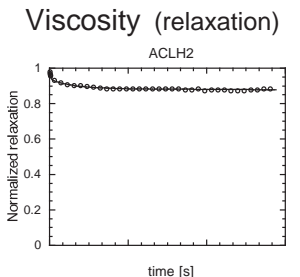

Long time memory

Figure 1. Uniaxial tension tests on human ACL. These tests highlight the different time scales of the mechanical behaviors. Immediate, short time memory and long time memory effects are present.

This approach would facilitate not only the comparison of viscoelastic properties between tissues but also the implementation of the constitutive law in a numerical model. To make the constitutive law as general as possible, it should be valid for large deformations and should satisfy the principles of thermodynamics. We present here the development of a general constitutive law describing, in the same conceptual framework, the immediate, the short and long time memory contributions. The experimental data are obtained from a PhD thesis Pioletti (1997)

\section{Time scale based viscoelastic constitutive law}

We start our description by considering the most general form for a constitutive law Truesdell and Noll (1992)

$$
\left.\boldsymbol{S}(t)=\boldsymbol{S}_{e}(\boldsymbol{C}(t))+\Im_{s=0}^{\infty}\{\boldsymbol{G}(t-s) ; \boldsymbol{C}(t))\right\},
$$

where $\boldsymbol{S}$ is the second Piola-Kirchhoff stress tensor, $\boldsymbol{C}$ is the right Cauchy-Green strain tensor, $\Im$ is a functional representing the history of $\boldsymbol{G}(t-s)=\boldsymbol{C}(t-s)-\boldsymbol{C}(t)$ and $\boldsymbol{S}_{e}(\boldsymbol{C}(t))$ is an equilibrium term. The stress $\boldsymbol{S}$ and strain $\boldsymbol{C}$ are two symmetric secondorder tensors.

The keypoint in the development of a constitutive law based on the time scale of the mechanical behaviors is to transform eq. (1) playing with its time interval Pioletti and Rakotomanan (2000)

$$
\left.\left.\boldsymbol{S}(t)=\boldsymbol{S}_{e}(\boldsymbol{C}(t))+\Im_{s=0}^{\delta}\{\boldsymbol{G}(t-s) ; \boldsymbol{C}(t))\right\}+\Im_{s=\delta}^{\infty}\{\boldsymbol{G}(t-s) ; \boldsymbol{C}(t))\right\},
$$

where $\delta \simeq 0$.The first term of the right hand-side of eq. (2) is the immediate contribution (elastic behavior) as it supports the contribution of the deformation at the actual time $t$. The second term of eq. (2) represents the short time memory effect while the third term represents the long time memory effect. It has been shown that eq. (2) can take the form Pioletti and Rakotomanan (2000) 


$$
\boldsymbol{S}(t)=\boldsymbol{S}_{e}(\boldsymbol{C}(t))+\boldsymbol{S}_{v}(\dot{\boldsymbol{C}}(t) ; \boldsymbol{C}(t))+\int_{\delta}^{\infty} \Sigma(\boldsymbol{G}(t-s), s ; \boldsymbol{C}(t)) d s
$$

The development of eq. (3) has the advantage of taking into account the different mechanical behaviors in one framework which facilitates the identification process. Moreover, as this law used objective stress and strain tensors, the frame invariance of the law is automatically satisfied. To be compliant with \index\{thermodynamical principles\}, the general constitutive law (3) is expressed in term of elastic and dissipative potentials Truesdell and Noll (1992) Germain (1986)

$$
\begin{aligned}
\boldsymbol{S}_{e}(t) & =2 \rho_{0} \frac{\partial W_{e}}{\partial \boldsymbol{C}} \quad \text { (immediate contribution), } \\
\boldsymbol{S}_{v}(t) & =2 \rho_{0} \frac{\partial W_{v}}{\partial \dot{\boldsymbol{C}}} \quad \text { (short time memory), } \\
\left(\boldsymbol{S}-2 \rho_{0} \mathbf{D} \aleph_{s=\delta}^{\infty}\{\boldsymbol{C}(t-s)\}\right): \frac{\dot{\boldsymbol{C}}}{2} & \geq 0 \quad \text { for all } \boldsymbol{C}, \dot{\boldsymbol{C}} \quad \text { (long time memory), }
\end{aligned}
$$

where $\mathbf{D}$ is a differential operator acting on the functional $\aleph$. For the long time memory, in the particular case where exponential relaxation functions are used, it can be demonstrated that the thermodynamic principles are satisfied Rabotnov (1977).

\section{Identification of the time scale based viscoelastic constitutive law}

Different elastic and viscous potentials can be tested in the general framework proposed until a satisfactory identification is obtained. In the present work, the identification is performed with uniaxial tension tests performed on human ACL Pioletti (1997). In order to obtain a tractable identification, the specimen are considered as isotropic, homogeneous and incompressible.

The potential for the immediate contribution is taken from Veronda and Westmann (1970)

$$
W_{e}=\frac{\alpha}{2} \exp \left[\beta\left(I_{1}-3\right)\right]-\frac{\alpha \beta}{2}\left(I_{2}-3\right),
$$

where $\alpha$ and $\beta$ are two elastic (immediate) parameters, $I_{1}=\operatorname{tr} \boldsymbol{C}$ and $I_{2}=1 / 2\left((\operatorname{tr} \boldsymbol{C})^{2}-\right.$ $\left.\operatorname{tr} C^{2}\right)$ are two invariants of the deformation tensor. Identification with the tension test performed at the lowest strain rate allowed us to determine the elastic parameters (see Figure 2).

The potential for the short term memory is taken from Pioletti et al. (1998)

$$
W_{v}=\frac{\eta^{\prime}}{4} \operatorname{tr}\left((\dot{\boldsymbol{C}})^{2}\right)\left(I_{1}-3\right)
$$

where $\eta^{\prime}$ is the short time memory parameter. Identification with tension tests performed at four different strain rates allowed us to determine the short term memory parameter 


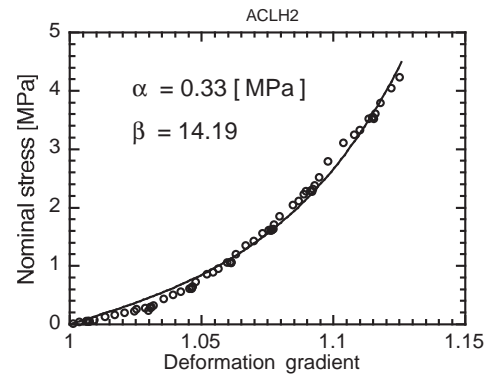

Figure 2. Experimental uniaxial tension test (dot) on human ACL and analytical results using the elastic potential (7).

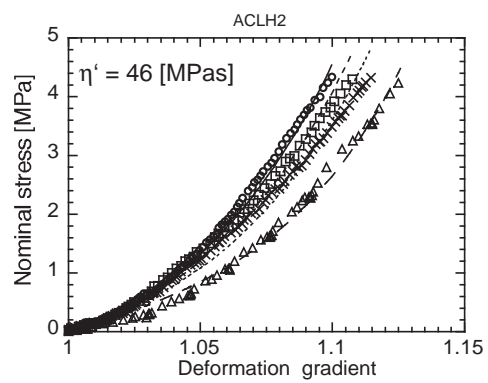

Figure 3. Experimental uniaxial tension tests (points) performed at 4 different strain rates on human ACL and analytical results (lines) using the viscous potential (8).

(see Figure 3).

Finally, for the long time memory, a normalized exponential Prony series is used Pioletti and Rakotomanan (2000)

$$
\int_{\delta}^{\infty} \Sigma(\boldsymbol{G}(t-s), s ; \boldsymbol{C}(t)) d s=\int_{\delta}^{t} \boldsymbol{S}_{e}(\boldsymbol{C}(t-s)) \dot{M}(s) d s
$$

with

$$
M(s)=\left(\sum_{k=1}^{3} a_{k} \exp \left(-\frac{s}{\tau_{k}}\right)\right) /\left(\sum_{k=1}^{3} a_{k}\right),
$$

This approach has been shown to satisfy thermodynamical principles as well as the principle of fading memory Coleman and Noll (1961). A possible set of six parameters $\left(a_{k}\right.$ and $\tau_{k}$ ) are identified with stress relaxation experiments (see Figure 4 ). It has to be noted that the choice of the six parameters is not unique and alternative sets of six parameters might equally well reproduce the curve.

In summary, the developed constitutive law taking into account immediate, short time memory and long time memory contributions takes the form 


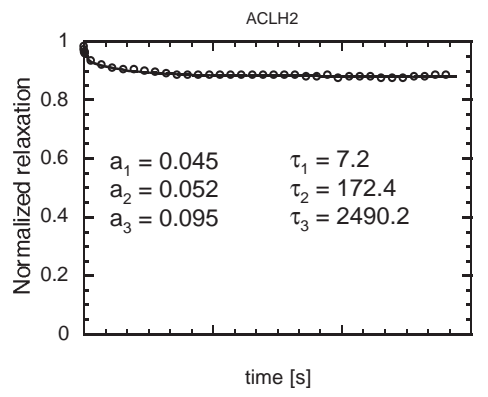

Figure 4. Experimental stress relaxation test (points) and analytical results (lines) using the Prony series (9 and 10).

$$
\begin{aligned}
\boldsymbol{S}_{e}(t) & =-p \boldsymbol{C}^{-1}+\alpha \beta\left(2 \exp \left[\beta\left(I_{1}-3\right)\right]-I_{1}\right) \boldsymbol{I}+\alpha \beta \boldsymbol{C}, \\
\boldsymbol{S}_{v}(t) & =\eta^{\prime}\left(I_{1}-3\right) \dot{\boldsymbol{C}}, \\
\int_{\delta}^{\infty} \Sigma(\boldsymbol{G}(t-s), s ; \boldsymbol{C}(t)) d s & =\int_{\delta}^{t} \boldsymbol{S}_{e}(\boldsymbol{C}(t-s)) \dot{M}(s) d s,
\end{aligned}
$$

The presence of the term $-p \boldsymbol{C}^{-1}$ is due to the kinematic constraint of incompressibility used.

\section{Advantages and limitations of the constitutive law based on time scale}

In the presented constitutive law, the main limitation is the isotropic assumption. It is well known that soft tissues such as ligaments present a transverse isotropic symmetry. However, the developed constitutive law based on time scale eq. (2) is general enough to incorporate different symmetries. The limitation to isotropy followed in this study was more due to the one-dimensional experimental identification. Indeed, performing mechanical tests in transversal direction of ligaments or tendons is a challenge. As highlighted in the identification process, the separation of the different mechanical behaviors according to their time scale is an advantageous process. It also facilitates the incorporation of resulting constitutive laws in numerical codes.

It has to be mentioned that the approach followed is phenomenological. This kind of description does then not furnish insight to the nature or origin of the viscoelastic phenomena in soft tissues. For such description, poro-elastic descriptions may be more appropriate. But it has to be clarified that finally any descriptions will be considered as phenomenological compared to a description taking more phenomena into account.

Probably the major advantage of the proposed description is to satisfy a priory objectivity and thermodynamical requirements. The developed description could then be used to model the viscoelastic behavior of different tissues such as bone or intervertebral discs. This represents the next step of our work. 


\section{References}

Palmer, E. I., and Lotz, J. C. (2004). The compressive creep properties of normal and degenerated murine intervertebral discs. J. Orthop. Res. 22:164-169.

Danto, M. I., Woo, S. L. (1993). The mechanical properties of skeletally mature rabbit anterior cruciate ligament and patellar tendon over a range of strain rates. J. Orthop. Res. 11:58-67.

Pioletti, D. P., Rakotomanna, L. R., Leyvraz, P. F. (1999). Strain rate effect on the mechanical behavior of the anterior cruciate ligament-bone complex. Med. Eng. Phys. 21:95-100.

Pioletti, D. P. (1997). Viscoelastic properties of soft tissues: application to knee ligaments and tendons. PhD thesis EPFL.

Truesdell, C., Noll, W. (1992). The Non-Linear Field Theories of Mechanics. SpringerVerlag.

Pioletti, D. P., and Rakotomanana, L. R. (2000). Non-linear viscoelastic laws for soft biological tissues. Eur. J. Mech. A/Solids 19:749-759.

Germain, P. (1986). Mecanique- Tome 1-2. Ellipses.

Rabotnov, Y. C. (1977). Elements of Hereditary Solid Mechanics. Mir.

Veronda, D. R., and Westmann, R. A. (1970). Mechanical consideration of skin-finite deformation. J. Biomechanics 3:111-124.

Pioletti, D. P., Rakotomanana, L. R., Benvenuti, J. F., Leyvraz, P. F. (1998). Viscoelastic constitutive law in large deformations: application to human knee ligaments and tendons. J. Biomechanics 31:753-757.

Coleman, B. D., Noll, W. (1961). Foundations of linear viscoelasticity. Rev. Modern Phys. 3:239-249. 